\title{
PROPIEDADES FÍSICO-MECÁNICAS DE UNA GUADUA MEXICANA (Guadua aculeata)
}

\section{PHYSICAL-MECHANICAL PROPERTIES OF A MEXICAN GUADUA (Guadua aculeata)}

\author{
Idalia Zaragoza-Hernández ${ }^{1}$, Víctor Rubén Ordóñez-Candelaria ${ }^{2}$, Guadalupe Martha Bárcenas- Pazos ${ }^{2}$, \\ Amparo Máxima Borja-de la Rosa ${ }^{1, \AA}$, Francisco José Zamudio-Sánchez
}

\section{RESUMEN}

En este artículo se presentan las propiedades físicas y mecánicas del culmo de Guadua aculeata. El material de estudio se colectó en el predio "Las Margaritas", Hueytamalco, Puebla, México. El marcado, derribo y ensayo se realizó tomando como referencia las normas ISO 22157-1 ISO e ISO/TR 22157-2. Se presentan valores promedio de los resultados para las secciones inferior, media y superior; se observa que la densidad básica (peso anhidro/volumen verde) varia de 560 a $660 \mathrm{~kg} / \mathrm{m}^{3}$ de la base a la parte superior del culmo. Las contracciones totales promedio fueron de $13,9 \%$ en espesor; $7,1 \%$ en diámetro y $0,1 \%$ en longitud. En el caso de las propiedades mecánicas se encontró que el esfuerzo máximo cortante varió de 6,0 a 10,5 MPa; el esfuerzo máximo en compresión paralela a la fibra incrementó de 28,2 a 56,7 MPa de la sección inferior a la superior y su módulo de elasticidad de 13,7 a 20,7 GPa. El módulo de ruptura en flexión de 51,9 a 79,6 MPa y su módulo de elasticidad de 15,1 a 24,1 GPa. La resistencia en tensión paralela a la fibra se incrementó de 58,5 a 92,2 MPa y el módulo de elasticidad en ese esfuerzo de 8,2 a 9,8 GPa. La especie estudiada tiene propiedades físicas, mecánicas y geométricas similares a la G. angustifolia que se utiliza en la construcción en países como Colombia, Costa Rica, Panamá, Venezuela y Ecuador.

Palabras claves: Compresión paralela, contracción longitudinal, contracción transversal, densidad básica, esfuerzo de corte, esfuerzo máximo, módulo de elasticidad, módulo de ruptura, tensión paralela.

\begin{abstract}
The physical and mechanical properties of Guadua aculeata culm are shown in this paper. The study material was collected from the locality "Las Margaritas" Hueytamalco, Puebla, Mexico. The marking, felling and testing was performed with reference to ISO standards ISO 22157-1 and ISO/TR 22157-2. The average values of the results of low, middle and upper sections are shown; the basic density (dry weight / green volume) ranged from 560 to 660 $\mathrm{kg} / \mathrm{m}^{3}$ of the lower to upper culm section. The average total contractions were $13,9 \%$ in thickness; $7,1 \%$ in diameter and $0,1 \%$ in length. In the case of mechanical properties were found that the maximum shear stress varies from 6,0 to $10,5 \mathrm{MPa}$; the maximum stress in compression parallel to fiber increased from 28,2 to $56,7 \mathrm{MPa}$ from low to top section and the modulus of elasticity from 13,7 to $20,7 \mathrm{GPa}$. The modulus of rupture in bending varies from 51,9 to $79,6 \mathrm{MPa}$ and the modulus of elasticity from 15,1 to $24,1 \mathrm{GPa}$. The strength in tension parallel to the fiber also increased from 58,5 to $92,2 \mathrm{MPa}$ and modulus of elasticity ranged from 8,2 to 9,8 $\mathrm{GPa}$. The studied specie has the same physical, mechanical and geometric properties to G. angustifolia that it is used in the construction in countries how Colombia, Costa Rica, Panama, Venezuela and Ecuador.
\end{abstract}

Keywords: Compression parallel, basic density, bending stress, longitudinal shrinkage, maximum stress, modulus of elasticity, modulus of rupture, tension parallel, transversal shrinkage.

\footnotetext{
${ }^{1}$ División de Ciencias Forestales. Universidad Autónoma Chapingo, Texcoco, Edo. de México, México

${ }^{2}$ Instituto de Ecología, A. C. Red de Ambiente y Sustentabilidad. Jalapa, Veracruz, México.

^Autor de correspondencia: aborja@correo.chapingo.mx

Recibido: 14.08.2013 Aceptado: 18.10.2014
} 


\section{INTRODUCCIÓN}

Los bambúes son un grupo de especies de rápido crecimiento y gran versatilidad. Su utilización va desde el consumo humano de sus brotes tiernos hasta el uso de tallos maduros en la construcción, fabricación de muebles, tableros, y otros productos industrializados. Un uso tradicional en México se da en la construcción de viviendas de las zonas rurales; actualmente, se están haciendo construcciones a mayor escala en las que es el elemento estructural principal, además, se usa en otros elementos de casas habitación y muebles (Ordóñez 1999, Cortés 2006).

Los culmos o tallos son relativamente fáciles de cortar, ligeros, de fácil manejo y resistentes. Esto hace del bambú un elemento útil, práctico y confiable para ser usado en aplicaciones donde la resistencia es un factor de alta consideración (Ordóñez 1999). La resistencia de las fibras del bambú a tensión, 503 MPa (Rao y Rao 2007), llegan a ser tan resistentes o más que el acero (385 a $400 \mathrm{MPa}$, Silva et al. 2008) o de otras fibras como las de palma real que tiene valores de $549 \mathrm{MPa}$ (Rao y Rao 2007).

La Guadua aculeata especie nativa de México, se ha usado de forma tradicional en la región norte de los estados de Veracruz y Puebla para paredes y travesaños de viviendas. El culmo se corta a la mitad longitudinalmente y se despliega golpeándolo hasta formar un tablero plano; uniendo los tableros a culmos enteros se obtienen paredes de buena calidad (Cortés 2005).

No obstante el potencial de la especie, su utilización ha sido sólo de manera empírica. La información tecnológica sobre los culmos de esta gramínea es mínima, incluyendo las propiedades físicas y mecánicas. Estas propiedades son básicas para el diseño y la construcción de elementos estructurales que con las medidas adecuadas se asegura la funcionalidad del material en una estructura (CONAFOR 2006, Londoño 2006).

Los objetivos del estudio fueron evaluar algunas propiedades físicas de culmos de G. aculeata como densidad básica, contenido de humedad y contracciones totales; y las propiedades mecánicas de flexión; cortante, compresión y tensión paralelos a la fibra.

\section{MATERIALES Y MÉTODOS}

La guadua (Guadua aculeata Rupr. ex Fournier), se recolectó en bambusal natural, en el predio "Las Margaritas" del municipio de Hueytamalco, Puebla; ubicado entre las coordenadas geográficas $19^{\circ} 52^{\prime}$ y $20^{\circ} 12^{\prime}$ de latitud norte y $97^{\circ} 12^{\prime}$ y $97^{\circ} 23^{\prime}$ de longitud oeste. El sitio de colecta está a una altitud de $430 \mathrm{msnm}$. La región presenta un clima cálido, húmedo con abundantes lluvias en verano. Es una zona de transición con áreas reducidas de selva alta perennifolia; con vegetación secundaria arbustiva y bosque mesófilo de montaña (INEGI 2009, INEGI 2013).

La recolecta de los culmos, la preparación de las probetas y la realización de los ensayos se realizó conforme a las normas ISO (2004a) e ISO (2004b), cada culmo se dividió en tres partes iguales identificándolas por su posición en inferior, media y superior, igualmente con base en la información de las normas se tomó una muestra de 12 culmos para ensayar en verde y 12 para ensayar en seco; con este tamaño de muestra se obtiene un $90 \%$ de confiabilidad en los resultados, ensayando cada una de las porciones se obtiene la variación de las propiedades con la altura y los ensayos en verde y en seco las diferencias generadas por el cambio del contenido de humedad. Se utilizaron los procedimientos de pruebas y cálculo de resultados del Laboratorio de pruebas de productos maderables del Instituto de Ecología, A.C.; en donde se realizaron los ensayos.

El material en verde se ensayó inmediatamente; la otra parte de la muestra se puso a secar al aire libre bajo techo por un periodo de tres meses, antes de elaborar las probetas. Los ensayos mecánicos realizados fueron flexión, cortante, compresión y tensión paralelas a la fibra utilizando una máquina universal de ensayos con capacidad de $250 \mathrm{kN}$, con software propio para calcular los resultados. Para cada espécimen ensayado se determinó su densidad básica $(\mathrm{Db})$ como la relación entre su peso anhidro (PA) y volumen verde (VV) como PA/VV; y su contenido de humedad $(\mathrm{CH})$ al momento del ensayo. También se cuantificaron las contracciones totales. Las comparaciones múltiples de medias se realizaron con pruebas de DUNCAN con un nivel de significancia del 5\% para los resultados de los ensayos. Así mismo, para conocer el grado de dispersión de los resultados se presentan los valores de desviación estándar (S). 


\section{RESULTADOS Y DISCUSIÓN}

Los resultados de las propiedades mecánicas se presentan para condición verde y seca. en las tablas que contienen los resultados se indican letras junto a los valores; estas son iguales cuando presentaron diferencia no significativa entre ellas, con un nivel de significancia del 5\%.

El bambú estudiado presentó un valor mínimo de densidad básica $(\mathrm{Db})$ de $370 \mathrm{~kg} / \mathrm{m}^{3}$ y el máximo de $920 \mathrm{~kg} /$ $\mathrm{m}^{3}$. En la tabla 1 se presentan los valores promedios de la densidad para las tres secciones en que se dividió el culmo. En cada tabla de las propiedades mecánicas se presentan valores de Db correspondientes a las probetas ensayadas para esa propiedad. En la tabla 2 se muestran valores mínimos y máximos encontrados por otros autores y de otras especies.

Tabla 1. Valores de densidad básica, para la G. aculeata de Hueytamalco, Puebla, determinados por sección del culmo.

\begin{tabular}{|c|c|c|c|}
\hline & \multicolumn{3}{|c|}{ Sección del culmo } \\
\hline Estadístico & Inferior & Media & Superior \\
\hline $\begin{array}{c}\text { Densidad Básica } \\
\text { promedio }\left(\mathrm{kg} / \mathrm{m}^{3}\right)\end{array}$ & 560 & 560 & 660 \\
\hline Desviación estándar & 59 & 58 & 81 \\
\hline Coef. variación $(\%)$ & 10 & 9,8 & 12,3 \\
\hline
\end{tabular}

Tabla 2. Valores de densidad básica de varias especies obtenidas de la bibliografía.

\begin{tabular}{|c|c|c|l|}
\hline Especie & $\begin{array}{c}\text { Db, mínima } \\
\left(\mathrm{kg} / \mathrm{m}^{3}\right)\end{array}$ & $\begin{array}{c}\text { Db, máxima } \\
\left(\mathrm{kg} / \mathrm{m}^{3}\right)\end{array}$ & \multicolumn{1}{|c|}{ Autor } \\
\hline Guadua aculeata & 370 & 920 & Presente estudio \\
\hline Varias & 500 & 900 & Liese 1987, Liese 1998 \\
\hline Guadua angustifolia & 620 & 900 & Correal y Arbeláez 2010 \\
\hline Dendrocalamus asper & 560 & 700 & Surjokusumo y Nugroho 1995 \\
\hline Bambusa heterostachya & 390 & 580 & Mohmod. et al. 1995 \\
\hline Bambusa vulgaris & 690 & 840 & Valero et al. 2005 \\
\hline Bambusa blumeana & 390 & 590 & Espiloy 1985 \\
\hline Chusquea culeou & 550 & 690 & Poblete et al. 2009 \\
\hline Gigantochloa levis & 470 & 800 & Nordahlia et al. 2012 \\
\hline
\end{tabular}

La variación tan amplia en la $\mathrm{Db}$ puede explicarse por las características anatómicas del bambú; esto aunado a la mayor heterogeneidad que hay en individuos provenientes de bosques naturales.

Las contracciones totales obtenidas para la G. aculeata (Tabla 3 ) tienen valores que varían de $8,1 \%$ a 5,5\% en el diámetro de $15,5 \%$ a $10,2 \%$ en el espesor de pared de la sección inferior a la sección superior pero se aprecia entre la parte inferior y la media muy poca diferencia entra los valores de contracciones, siendo más marcada la diferencia contra los valores de contracciones para la sección superior y los de la parte inferior sin existir diferencia significativa. Los resultados son comparables a los encontrados en la bibliografía; como los de G. angustifolia (Sotela 1992) de 6,7 \% a 6,9 \% en el diámetro de la variedad Atlántico y de 23,8 \% a 14,0 \% en el espesor para la misma variedad; y de 8,9\% a 6,2 \% en el diámetro de la variedad Sur y $18,6 \%$ a $15,8 \%$ en el grosor para la misma variedad. Para $B$. heterostachya de 3 años de edad, Mohmod. et al. (1995), obtuvo valores de 8,9 \% a 7,6 \% en el diámetro y de 18,9 \% a 19,2 \% en el grosor. Esta propiedad debe tomarse en cuenta en el proceso de secado y en la elaboración de cualquier producto con el fin de reducir los defectos generados por el proceso, principalmente las grietas y rajaduras. 
Tabla 3. Valores promedio de contracciones totales a tres alturas del culmo de G. aculeata.

\begin{tabular}{cccc}
\hline \multirow{2}{*}{ Sección } & \multicolumn{3}{c}{ Contracciones promedio (\%) } \\
\cline { 2 - 4 } & Espesor & Diámetro & Longitud \\
\hline${\text { Inferior }(570)^{\mathrm{a}}}^{\mathrm{a}}$ & $15,5 \mathrm{~A}$ & $8,1 \mathrm{~B}$ & $0,1 \mathrm{C}$ \\
${\text { Media }(580)^{\mathrm{a}}}^{\mathrm{a}}$ & $15,9 \mathrm{~A}$ & $7,8 \mathrm{~B}$ & $0,1 \mathrm{C}$ \\
${\text { Superior }(690)^{\mathrm{a}}}^{\mathrm{a}}$ & $10,2 \mathrm{~A}$ & $5,5 \mathrm{~B}$ & $0,1 \mathrm{C}$ \\
Promedio & 13,9 & 7,1 & 0,1 \\
\hline
\end{tabular}

${ }^{\mathrm{a}}$ Densidad básica, $\mathrm{Db}\left(\mathrm{kg} / \mathrm{m}^{3}\right)$

Los valores de resistencia en cortante, $f v_{\max x}$ que se obtuvieron para la $G$. aculeata se presenta en la tabla $4 \mathrm{y}$ se observa que no hay gran diferencia entre las secciones inferior y media pero si hay un incremento en la sección superior, tanto en seco como en verde y este comportamiento se presenta para los especímenes con nudo y sin nudo, la relación entre la resistencia y la $\mathrm{Db}$ así como el efecto del $\mathrm{CH}$ se muestra en las gráficas de la figura 1 y 2. Los resultados son parecidos a los encontrados en la bibliografía por otros autores como los encontrados para G. angustifolia que varían de 7,1 MPa a 7,8 MPa en verde (Correal y Arbeláez 2010), en G. angustifolia cebolla de 5,57 MPa (Ciro et al. 2005), en G. angustifolia atlántica de 6,18 MPa y en G. angustifolia sur de 7,35 MPa (Sotela 1992), en Gigantochloa scortechinii de 4,1 MPa a 6,8 MPa (Bahari y Hamad 2009), en C. culeou de 9,09 MPa (Poblete et al. 2009) y en D. asper de 11,20 MPa a 12,52 MPa (Surjokusumo y Nugroho 1995), los últimos seis casos en condición seca. En general, el $f v_{\text {máx }}$ incrementó con una mayor densidad básica como se observa en las figura 1 y 2. Otro factor que mostró cierta influencia positiva fue la ausencia del nudo. Los ensayos en seco presentan valores similares en las dos primeras secciones del culmo pero se incrementa ligeramente en la sección superior, tanto en muestras con nudo y sin nudo (Tabla 4, Figura 1 y 2 ).

Tabla 4. Esfuerzo máximo en cortante paralelo $\left(f v_{\max x}, M P a\right)$ a la dirección de la fibras, en condiciones verde y seca de G. aculeata de Hueytamalco, Puebla.

\begin{tabular}{|c|c|c|c|}
\hline & \multicolumn{3}{|c|}{ Sección del culmo } \\
\hline Estadístico & Inferior & Media & Superior \\
\hline $\begin{array}{c}\text { Densidad Básica } \\
\text { promedio }\left(\mathrm{kg} / \mathrm{m}^{3}\right)\end{array}$ & 560 & 560 & 660 \\
\hline Desviación estándar & 59 & 58 & 81 \\
\hline Coef. variación(\%) & 10 & 9,8 & 12,3 \\
\hline
\end{tabular}




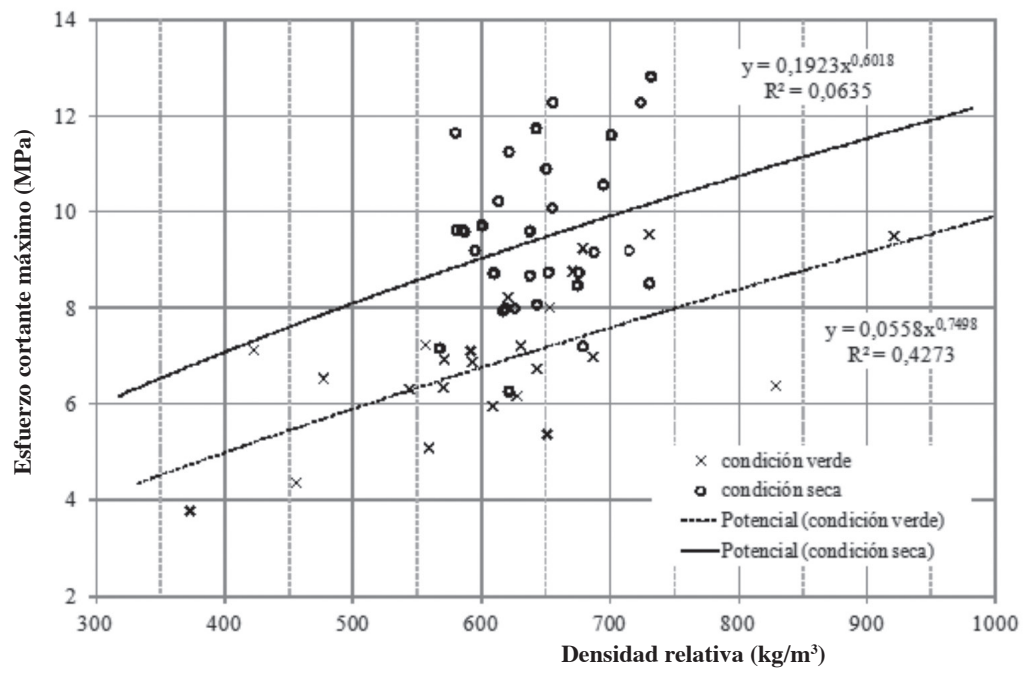

Figura 1. Relación entre densidad básica y esfuerzo máximo cortante en probetas sin nudo, en condiciones verde y seca de G. aculeata de Hueytamalco, Puebla.

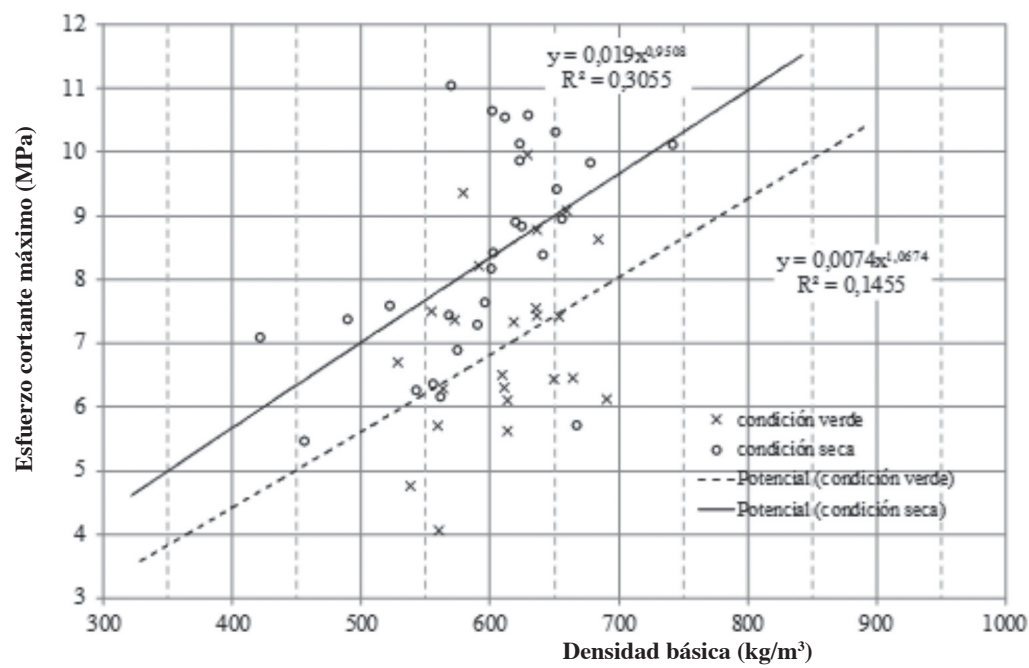

Figura 2. Relación entre densidad básica $(D b)$ y esfuerzo máximo cortante $\left(f v_{\max }\right)$ en probetas con nudo, en condiciones verde y seca de G. aculeata de Hueytamalco, Puebla.

La resistencia máxima $\left(f c_{\text {max }}\right)$ y módulo de elasticidad $(E c)$ en compresión paralela a la fibra se incrementan con la altura, pero en donde se presenta un mayor incremento es entre la condición seca sobre la verde, de hasta el 69 $\%$ de 28,3 MPa a 47,9 MPa (tabla 5); y el incremento en el valor del módulo de elasticidad fue del $41 \%$ de 13,7 MPa a 19,3 MPa (tabla 5) ambos en la porción inferior, esto se observa claramente en las gráficas de las figura 3 y 4 que presentan la correlación entre $\mathrm{Db}$ y la resistencia a compresión. Los valores encontrados en la bibliografía para $f c_{\text {mix }}$ de $G$. angustifolia Var. Macana en seco varían en la altura de 50,6 MPa a 59,7 MPa (Takeuchi y González 2007); en otro estudio sobre G. angustifolia su resistencia va de 37,6 MPa a 42,1 MPa entre la parte inferior y la superior en seco para edad de cuatro años (Correal y Arbeláez 2010); otros valores promedio para G. angustifolia Atlántico de 41,8 MPa en seco y para G. angustifolia Sur de 41,2 MPa en seco (Sotela 1992); para la especie de B. blumeana su resistencia varía de 25,3 MPa a 28,9 MPa de la parte inferior a la superior (Latif et al. 1992). El CH del culmo fue en promedio $21 \%$, por ello se supone un aumento importante del $f c_{\text {max }}$ con una disminución mayor del $\mathrm{CH}$; esto podría superar los valores de varias especies incluyendo a $G$. angustifolia. 
Tabla 5. Esfuerzo máximo $\left(f c_{\max x}, M P a\right)$ y módulo de elasticidad $(E c, G P a)$ en compresión paralela a la fibra, en condiciones verde y seca de G. aculeata de Hueytamalco, Puebla.

\begin{tabular}{cccccccc}
\hline & \multicolumn{7}{c}{ Sección del culmo } \\
\cline { 2 - 7 } & \multicolumn{2}{c}{ Inferior } & \multicolumn{2}{c}{ Media } & \multicolumn{2}{c}{ Superior } \\
\hline \multicolumn{2}{l}{ Condición } & verde & seco & Verde & seco & verde & seco \\
\hline \multirow{2}{*}{$\mathrm{fc}_{\text {máx }}$} & $\mathrm{X}$ & $28,3 \mathrm{D}$ & $47,9 \mathrm{~B}$ & $28,9 \mathrm{D}$ & $50,9 \mathrm{~B}$ & $35,2 \mathrm{C}$ & $56,6 \mathrm{~A}$ \\
& $\mathrm{~S}$ & 3,8 & 6,6 & 2,9 & 4,4 & 4,9 & 2,9 \\
& $\mathrm{Db}$ & 569 & 602 & 578 & 605 & 649 & 675 \\
\hline \multirow{2}{*}{$\mathrm{Ec}$} & $\mathrm{X}$ & $13,7 \mathrm{~B}$ & $19,3 \mathrm{~A}$ & $16,1 \mathrm{~B}$ & $20,7 \mathrm{~A}$ & $18,0 \mathrm{~A}$ & $19,7 \mathrm{~A}$ \\
& $\mathrm{~S}$ & 2,4 & 4,8 & 4,6 & 3,5 & 3,8 & 2,2 \\
& $\mathrm{Db}$ & 569 & 602 & 578 & 605 & 649 & 675 \\
\hline
\end{tabular}

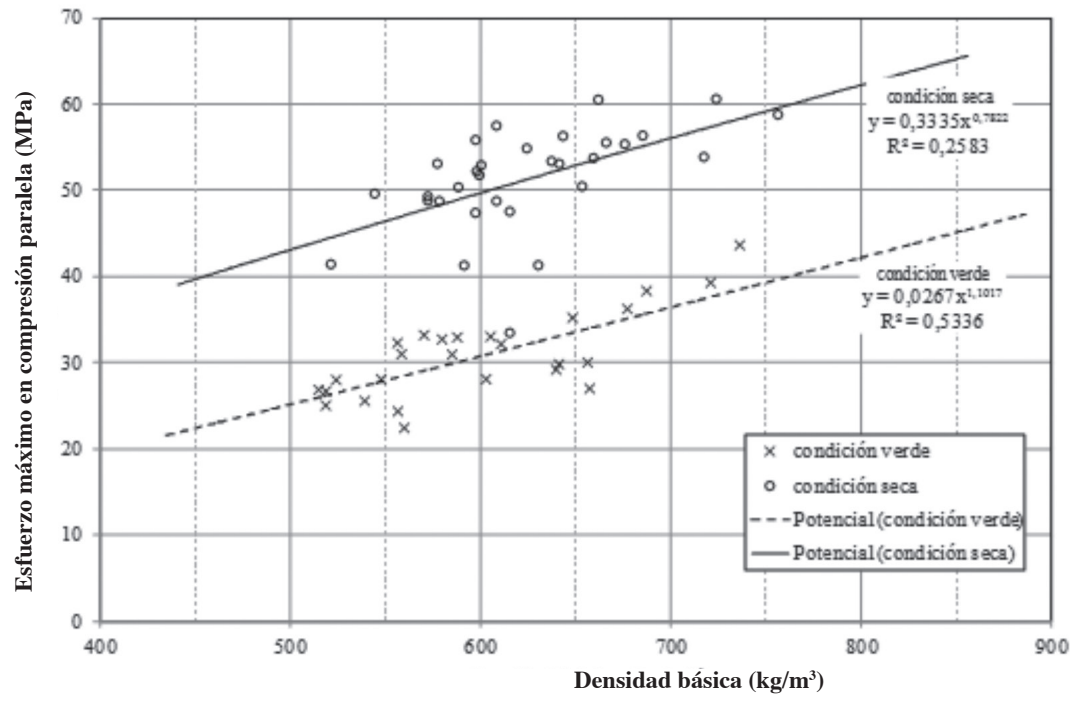

Figura 3. Relación entre densidad básica $(D b)$ y esfuerzo máximo en compresión ( $f c m a ́ x)$, en condiciones verde y seca de G. aculeata de Hueytamalco, Puebla.

Y en el caso de módulo de elasticidad $(E c)$ los valores encontrados por otros autores para $G$. angustifolia Var. Macana estuvieron entre 17,4 GPa y 18,1 GPa en seco (Takeuchi y González 2007); para G. angustifolia atlántico 18,9 GPa y para G. angustifolia. Sur 17,3 GPa en seco (Sotela 1992); otro más de G. angustifolia varió de 14,9 GPa a $20 \mathrm{GPa}$ en verde (Correal y Arbeláez 2010). El Ec de G. aculeata aumentó de la base hacia la zona apical hasta en $34 \%$ en condición verde; en seco no se presenta tal variación, situación similar a $G$. angustifolia de la bibliografía. $\mathrm{El}$ aumento de $E c$ al bajar el $\mathrm{CH}$ estuvo entre el 9 y 41 \%, siendo mayor en la sección inferior (Tabla 5 y Figura 4). 


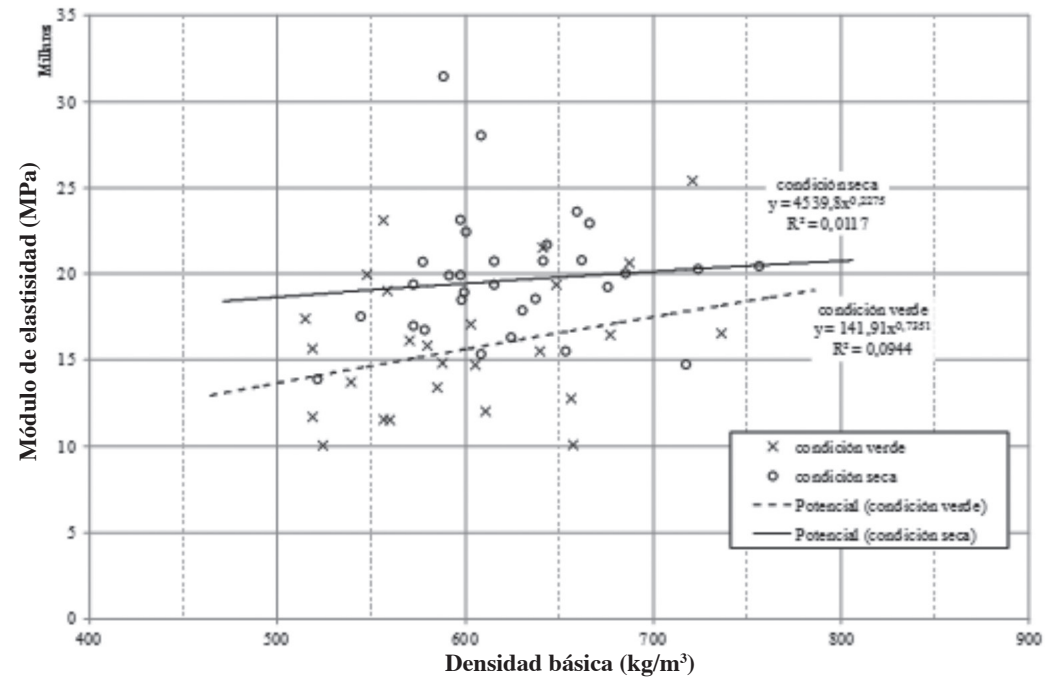

Figura 4. Relación entre densidad básica $(D b)$ y módulo de elasticidad en compresión $(E c)$, en condiciones verde y seca de G. aculeata de Hueytamalco, Puebla.

Los resultados en flexión de la $G$. aculeata estudiada tiene un comportamiento que difiere de las demás propiedades, los valores de MOR (tabla 6) se incrementan de la parte inferior y la superior pero la parte media es menor que la inferior en verde $61,6 \mathrm{MPa} ; 51,9 \mathrm{MPa}$ y 69,5 MPa; pero en seco la parte inferior resultó más resistente que la parte media y la superior 79,6 MPa; $62,8 \mathrm{MPa}$ y 73,3 MPa; de la misma manera, los valores del MOE varían en verde de 16,7 GPa; 15,1 GPa y 18,6 GPa; y en seco 21,3 MPa; 20,8 GPa y 24,1 GPa. Correal y Arbeláez (2010) estudiaron G. angustifolia y obtuvieron valores para MOR de 86,9 MPa; 103,7 MPa y 103,8 MPa, para las secciones inferior, media y superior respectivamente en condición verde para culmos con cuatro años de edad; en otro trabajo con Bambusa vulgaris (Valero et al. 2005) se encontraron valores de 111,3 MPa; 81,5 MPa y 64,1 MPa para MOR de ensayos con tiras en condición verde y 136,2 MPa; 104,3 MPa y 66,1 MPa para ensayos en condición seca. En el caso de MOE igual que para MOR cuando se presenten tres valores corresponden a las secciones inferior, media y superior, así para G. angustifolia (Correal y Arbeláez 2010) presenta valores de 17,0 GPa; 17,0 GPa y 18,3 GPa en verde, estos valores son similares a los obtenidos en este trabajo; otros datos encontrados para G. angustifolia (Gnanaharan et al. 1994) son de 72,6 MPa para el MOR y de 17,6 GPa para MOE en donde no diferenciaron las secciones del culmo; otra de las especies que se han estudiado es el Dendrocalamus asper (Subyakto 1995) haciendo ensayos con tiras encontró valores para MOE de 4,4 Gpa; 4,6 GPa y 3,4 GPa en condición seca, valores menores a los encontrado para la G. aculeata. 
Tabla 6. Módulo de ruptura ( $M O R, M P a)$ y módulo de elasticidad ( $M O E, G P a)$ en flexión estática, en condiciones verde y seca de G. aculeata de Hueytamalco, Puebla.

\begin{tabular}{|c|c|c|c|c|c|c|c|}
\hline & & \multicolumn{6}{|c|}{ Sección del culmo } \\
\hline & & \multicolumn{2}{|c|}{ Inferior } & \multicolumn{2}{|c|}{ Media } & \multicolumn{2}{|c|}{ Superior } \\
\hline & & \multicolumn{2}{|c|}{ seco } & \multicolumn{2}{|c|}{ seco } & \multicolumn{2}{|c|}{ seco } \\
\hline & & verde & & verde & & verde & \\
\hline \multirow{3}{*}{ MOR } & $\mathrm{X}$ & $61,6 \mathrm{~B}$ & $79,6 \mathrm{~A}$ & $51,9 \mathrm{~B}$ & $62,8 \mathrm{~B}$ & $69,5 \mathrm{~A}$ & $73,3 \mathrm{~A}$ \\
\hline & $S$ & 10,8 & 19,6 & 13 & 11,6 & 9,5 & 13,9 \\
\hline & $D b$ & 554 & 604 & 565 & 605 & 650 & 663 \\
\hline \multirow{3}{*}{$\mathrm{MOE}$} & $\mathrm{X}$ & $16,7 \mathrm{~B}$ & $21,3 \mathrm{~A}$ & $15,1 \mathrm{~B}$ & $20,8 \mathrm{~A}$ & $18,6 \mathrm{~B}$ & $24,1 \mathrm{~A}$ \\
\hline & $S$ & 4,3 & 4,9 & 5,8 & 6,3 & 3,9 & 5,3 \\
\hline & $D b$ & 554 & 604 & 565 & 605 & 650 & 663 \\
\hline
\end{tabular}

En ambas condiciones de humedad se muestra la relación directa entre el incremento del $M O R$ y el $M O E$ conforme aumenta la densidad básica del bambú, es de resaltar el valor bajo de $\mathrm{R}^{2}$ para la condición seca debido a los defectos generados por el proceso de secado como son grietas y deformaciones del culmo (Figura 5 y 6 ).

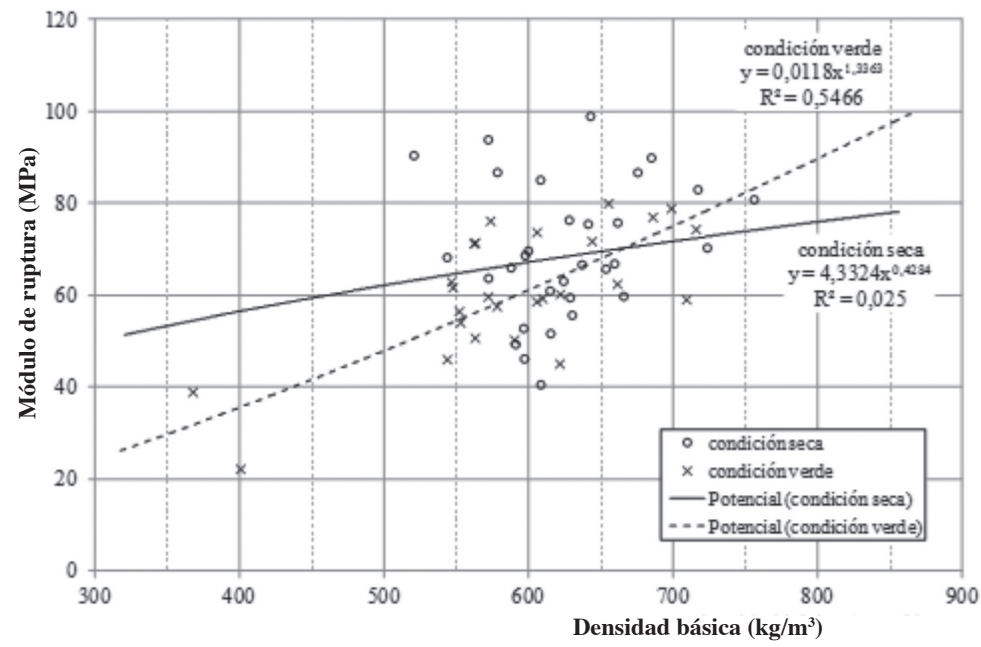

Figura 5. Relación de la densidad básica $(D b)$ y módulo de ruptura en flexión estática $(M O R)$, en condiciones verde y seca de G. aculeata de Hueytamalco, Puebla. 


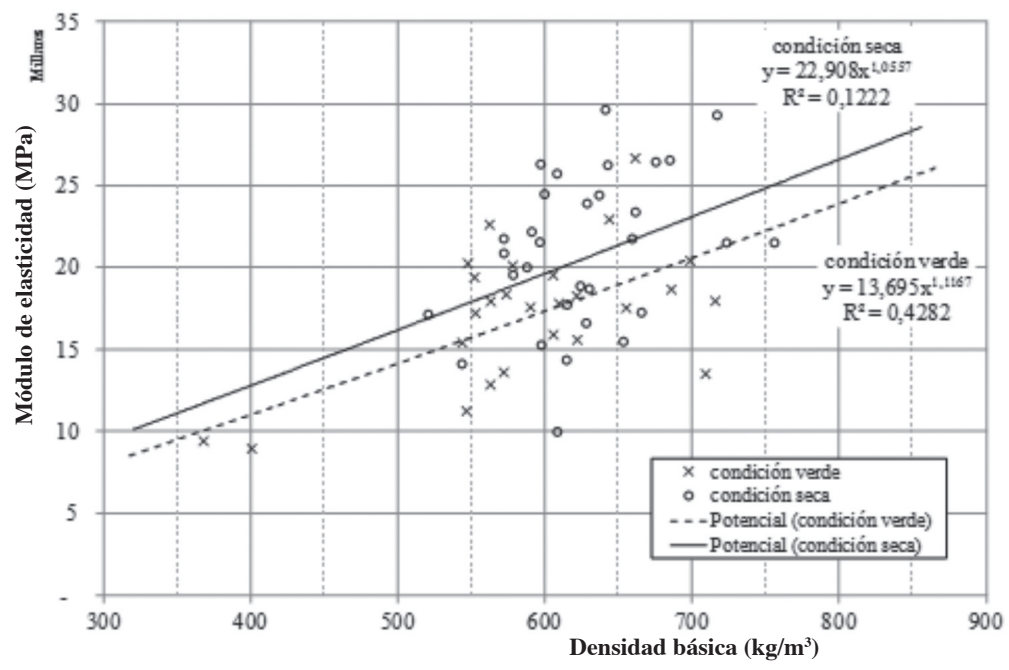

Figura 6. Relación densidad básica $(D b)$ y módulo de elasticidad en flexión estática $(M O E)$, en condiciones verde y seca de G. aculeata de Hueytamalco, Puebla.

La resistencia en tensión $\left(f t_{\text {max }}\right)$ de la guadua estudiada presenta valores de 58,5 MPa; $92,2 \mathrm{MPa}$ y 63,6 MPa (tabla 7) para las porciones inferior, media y superior del culmo y para el módulo de elasticidad (Et) 8,2 Gpa; 9,8 Gpa y 8,4 GPa para las mismas porciones. De la bibliografía se encontró para $B$. vulgaris (Valero et al. 2005) valores de $f t_{\text {max }}$ de $42 \mathrm{MPa}, 30 \mathrm{MPa}$ y 19,1 MPa en condición verde para las secciones ya mencionadas, en condición seca los valores son de 52,5 MPa, 40,6 MPa y 30,4 MPa; para Dendrocalamus asper (Surjokusumo y Nugroho 1995) encontró valores de $138 \mathrm{MPa}$ ensayando tiras con nudo como en el presente trabajo y 220,3 MPa cuando uso tiras sin nudo, ambas en condición seca; Ciro et al. 2005, encontró para G. angustifolia valores de 190,7 MPa sin diferenciar entre secciones, estos valores superan de manera importante los resultados de este trabajo; otros datos de G. angustifolia (González et al. 2006) con 154,8 MPa 187,7 MPa y 164,9 MPa para un Ch $\approx 15 \%$ correspondientes a las secciones inferior, media y superior, respectivamente de ensayos con tiras sin nudo. Como se puede ver la resistencia y rigidez de las guaduas es mayor en la sección media del culmo y se reduce para la inferior y superior, los valores menores de la G. aculeata con respecto a la $G$. angustifolia puede atribuirse a que se ensayaron tiras con nudos.

Tabla 7. Esfuerzo máximo $\left(f t_{\max }, M P a\right)$ y módulo de elasticidad en tensión $(E t, G P a)$ paralela a la fibra, en condición seca de G. aculeata de Hueytamalco, Puebla.

\begin{tabular}{cccc}
\hline & \multicolumn{3}{c}{ Sección del culmo } \\
\cline { 2 - 4 } & Inferior & Media & Superior \\
\hline$f t_{\text {máx }}$ & $58,5 \mathrm{~B}$ & $92,2 \mathrm{~A}$ & $63,6 \mathrm{~B}$ \\
$S$ & 12,4 & 28,2 & 7,6 \\
$D b$ & 590 & 560 & 620 \\
$E t$ & $8,2 \mathrm{~A}$ & $9,8 \mathrm{~A}$ & $8,4 \mathrm{~A}$ \\
$S$ & 1,4 & 3,5 & 2,2 \\
$D b$ & 590 & 560 & 620 \\
\hline
\end{tabular}




\section{CONCLUSIONES}

Como en la mayoría de las especies de bambú la densidad básica de la G. aculeata estudiada se incrementan de la base hacia el ápice del culmo, la sección superior presentó diferencias significativas con la sección inferior y media, no así entre la inferior y la media. Por el contrario las contracciones muestran una tendencia a ir reduciéndose hacia la porción superior.

Los resultados de resistencia en cortante $\left(f v_{\text {max }}\right)$ muestran un incremento de la sección inferior y media hacia la superior del culmo, en condición verde. Los especímenes en seco con nudo y sin nudo no presentan diferencias significativas entre sus tres secciones.

La resistencia en compresión paralela a la fibra $\left(f c_{\text {max }}\right)$ mostró un incremento del extremo superior sobre la sección inferior y media, se registraron diferencias significativas entre la sección superior y las otras dos secciones en ambas condiciones de humedad. El $E c$ en seco fue mayor que en estado verde y no presentó diferencias significativas entre sus tres secciones.

El valor más alto de la resistencia en flexión estática (MOR) en verde, se obtuvo en la sección superior del culmo, valores que difieren significativamente de las otras dos secciones. En condición seca el valor de MOR más bajo se obtuvo para los especímenes de la sección media y difiere de manera significativa de los extremos. El módulo de elasticidad en flexión (MOE) mayor lo presentaron las probetas secas, difieren significativamente de las verdes. Los promedios obtenidos de esfuerzo máximo $\left(f t_{\text {max }}\right)$ y módulo de elasticidad $(E t)$ en tensión paralela a la fibra fueron más altos en la parte media del culmo, sólo se presentaron diferencias significativas entre las zonas media y extremos del culmo.

Los resultados de este trabajo, permiten afirmar que los culmos de la G. aculeata son un material estructural que se puede usar con éxito en la construcción de estructuras tanto como la G. angustifolia usada ampliamente en Colombia país que tiene un gran desarrollo con este material. Es necesario realizar estudios sobre la durabilidad y sistemas de preservación para usar la G. aculeata con más probabilidades de éxito. 


\section{BIBLIOGRAFÍA}

Mohmod, A.L.; Amin, A.H.; Kasim, J.; Jusuh, M.Z. 1993. Effects of anatomical characteristics on the physical and mechanical properties of Bambusa blumeana. Journal of Tropical Forest Science 6(2):159-170.

Mohmod, A.L.; Amin, A.H.; Kasim, J.; Jusuh, M.Z. 1995. Chemical constituents and physical properties of Bambusa heterostachya. Proceedings of the Vth International Bamboo Workshop and the IV International Bamboo Congress. Indonesia. pp.225-238.

Bahari, S.A.; Ahmad, M. 2009. Effects of culm height levels and node presence on mechanical properties and fracture modes of Gigantochloa scortechinii strips loaded in shear parallel to grain. Bamboo Science and Culture: The Journal of the American Bamboo Society 22(1):41-44.

Ciro, H.J.; Osorio, J.A.; Vélez, J.M. 2005. Determinación de la resistencia mecánica a tensión y cizalladura de la Guadua angustifolia Kunth. Revista Facultad Nacional de Agronomía, Medellin 58(1):2709-2715.

Comisión Nacional Forestal. CONAFOR. 2006. El Bambú: tan fuerte como el acero. Revista electrónica de la Comisión Nacional Forestal

Correal, J.F.; Arbeláez, J. 2010. Influence of age and height position on colombian Guadua angustifolia bamboo mechanical properties. Maderas: Ciencia y Tecnología 12(2):105-113.

Cortés, G.R. 2005. Viviendas de bambú en México. Revista electrónica Bio bambú.

Cortés, G.R. 2006. El Bambú en México: la gente, la planta y el ambiente. Revista electrónica Bio Bambú.

Espiloy, Z.B. 1985. Physico-mechanical properties and anatomical relationships of some Philippine bamboos. International Bamboo Workshop. China. pp 257-264.

Gnanaharan, R.; Janssen, J.J.A.; Arce, O. 1994. Bending strength of Guadua bamboo. Comparison of different testing procedures. INBAR Working Paper No. 3. Dehli, India.

González, H.A; Montoya, J.A.; Bedoya, J.R. 2006. Esfuerzo de tensión y la influencia de la humedad relativa del ambiente y la altura a lo largo del tramo en la especie de bambú Guadua angustifolia Kunth. Scientia et Technica 12(32):445-450.

Instituto Nacional de Estadística y Geografía. INEGI. 2009. Prontuario de información geográfica municipal de los Estados Unidos Mexicanos.

Instituto Nacional de Estadística y Geografía (INEGI). 2013. Principales tipos de vegetación de México.

International Organization for Standardization. ISO. 2004a. Bamboo-Determination of physical and mechanical properties - Part 1: Requirements. ISO 22157-1. Switzerland. 23p.

International Organization for Standardization. ISO. 2004b. Bamboo-Determination of physical and mechanical properties - Part 2: Laboratory manual. ISO/TR 22157-2. Switzerland. First edition. 23p.

Liese, W. 1987. Research on bamboo. Wood Science and Technology 21:189-209.

Liese, W. 1998. The Anatomy of Bamboo Culms. INBAR Technical Report $\mathrm{N}^{\circ}$ 18. International Network for Bamboo and Rattan, Beijing. 204p.

Londoño, X. 2006. Aspectos generales de los bambúes americanos. Revista electrónica Bio Bambú.

Ordóñez, V.R. 1999. Perspectivas del bambú para la construcción en México. Madera y Bosques 5(1): 3-12. 
Poblete, H.; Cuevas, H.; Díaz-Vaz, J.E. 2009. Property characterization of Chusquea culeou, a bomboo growing in Chile. Maderas: Ciencia y Tecnología 11(2):129-138.

Rao, K.M.M; Rao, K.M. 2007. Extraction and tensile properties of natural fibers: vakka, date and bamboo. Composite Structures 77:288-295.

Sotela, J. 1992. El bambú: una alternativa de uso en construcción de vivienda. Ingeniería Revista de Universidad de Costa Rica 2(1):87-94.

Silva, F.A.; Chawla, N.; Toledo Filho, R.D. 2008. Tensile behavior of high performance natural (sisal) fibers. Composites Science and Technology 68: 3438-3443.

Subyakto. 1995. Variation in specific gravity and bending properties of Dendrocalamus asper culm grown in Bogor. Proceedings of the Vth International Bamboo Workshop and the IV International Bamboo Congress. pp. 185-192.

Surjokusumo, S.; Nugroho, N. 1995. A study on Dendrocalamus asper as concrete reinforcement. Proceedings of the Vth International Bamboo Workshop and the IV International Bamboo Congress. Indonesia. pp. 92-98.

Takeuchi, C.P.; González, C.E. 2007. Resistencia a la compresión paralela a la fibra de la Guadua angustifolia y determinación del módulo de elasticidad. Ingeniería y Universidad 11(001): 89-103.

Valero, S.W.; Reyes, E.; Contreras, W. 2005. Estudio de las propiedades mecánicas del bambú (Bambusa vulgaris), de tres años de edad y proveniente de las plantaciones ubicadas en la ribera de la margen derecha del rio Chama, municipio Francisco Javier Pulgar, estado Zulia, Venezuela. Revista Forestal Latinoamericana 37:87-107. 